\section{Case Reports in Ophthalmology}

Case Rep Ophthalmol 2020;11:342-347

DOI: $10.1159 / 000508821$

Published online: July 14, 2020
(C) 2020 The Author(s)

Published by S. Karger AG, Basel www.karger.com/cop

This article is licensed under the Creative Commons Attribution-NonCommercial 4.0 International License (CC BY-NC) (http://www.karger.com/Services/OpenAccessLicense). Usage and distribution for commercial purposes requires written permission.

\title{
Full-Thickness Macular Hole with Coats Disease: A Case Report
}

\author{
Yuto Hashimoto Yusuke Arai Shinji Makino Yuji Inoue \\ Hidenori Takahashi Hidetoshi Kawashima \\ Department of Ophthalmology, Jichi Medical University, Shimotsuke-shi, Japan
}

\section{Keywords}

Coats disease $\cdot$ Macular hole $\cdot$ Vitrectomy

\begin{abstract}
We report a case of full-thickness macular hole (FTMH) which developed during follow-up for Coats disease. To our knowledge, this is the first report on a case of FTMH which developed during follow-up for Coats disease. A 17-year-old boy was referred to our institution with blurred vision in his left eye. Fundus examination showed yellowish subretinal exudates with overlying telangiectatic retinal vessels in the temporal periphery in the left eye; the right eye was normal. Fluorescein angiography revealed diffusion indicative of temporal peripheral vascular leakage. We made a diagnosis of stage 2A Coats disease and performed argon laser photocoagulation (PC). Moreover, he received intravitreal injection of anti-vascular endothelial growth factor. At 30 months after the first visit, the best-corrected visual acuity (BCVA) of the left eye worsened to 20/50. We observed tensioned internal limiting membrane (ILM), serous macular detachment (SMD) and retinal thinning; the intraretinal exudate remained. We performed PC and at 36 months after the first visit, BCVA further declined to 20/63 in his left eye and FTMH was observed. We then performed a 27 -gauge pars plana vitrectomy and ILM peeling following which the FTMH was found to have closed by 4 days after vitrectomy. SMD gradually decreased, and BCVA improved to 20/20. Chronic inflammation by peripheral vascular leakage and PC application might have caused FTMH. In this case, the vitrectomy for FTMH with Coats disease provided good visual and anatomic outcomes.
\end{abstract}




\section{Case Reports in Ophthalmology}

\section{Introduction}

Coats disease is characterized by the occurrence of abnormal retinal telangiectasia with massive intraretinal and subretinal exudates of fluid and lipid, capillary nonperfusion, and exudative retinal detachment [1]. Coats disease presents with many clinical features and complications [2]; however, the development of full-thickness macular hole (FTMH) in Coats disease is particularly rare [3-6]. The etiology of FTMH in Coats disease is unknown. Here, we report on our case of a patient with Coats disease who developed FTMH during follow-up and discuss the pathogenesis of the FTMH.

\section{Case Report}

A 17-year-old boy complained of blurred vision in his left eye. Best-corrected visual acuity (BCVA) was 20/20 in both eyes. On fundus examination, the right eye was normal and the left eye had a large area of yellowish subretinal exudate with overlying telangiectatic retinal vessels in the temporal periphery. Fluorescein angiography revealed diffuse temporal peripheral vascular leakage and staining corresponding to retinal aneurysms (Fig. 1). Optical coherence tomography (OCT) showed retinal deposits, highly indicative of internal limiting membrane (ILM), and no sign of exudate (Fig. 2a). These clinical features led to a diagnosis of stage 2A Coats disease. Argon laser photocoagulation (PC) was performed for the temporal aneurysms, a total of 3 times, following which the subretinal exudate improved. This patient visited the United States to study and there he received intravitreal anti-vascular endothelial growth factor (VEGF) injection twice. At 30 months after the first visit, BCVA in his left eye declined to 20/50. Tensioned ILM, serous macular detachment (SMD) and retinal thinning was confirmed on OCT (Fig. 2b). Fundus examination showed that the intraretinal exudate was still present in the left eye. We performed additional PC and 36 months after the first visit, BCVA in his left eye further declined to 20/63. OCT revealed FTMH (Fig. 2c). The horizontal diameter of the macular hole was $262 \mu \mathrm{m}$. We then performed 27-gauge pars plana vitrectomy. There was no posterior vitreous detachment, and so we created it surgically. ILM peeling was performed using Brilliant Blue-G dye (0.06\%). Additional PC was performed for the peripheral retinal aneurysms. This was followed by tamponade with $20 \%$ sulfur hexafluoride gas. The patient was advised to maintain a face-down position until the FTMH closed. One day later, the FTMH remained. Four days after vitrectomy, the FTMH was found to be closed (Fig. 3a). OCT 1 month after vitrectomy showed SMD, but the FTMH remained closed (Fig. 3b). Follow-up at 6 months revealed that the subretinal exudate had gradually disappeared (Fig. 3c). BCVA improved to 20/20 and SMD decreased (Fig. 3d). Postoperatively, there was no progression of cataract and recurrence of intraocular inflammation during the 24 months of follow-up period.

\section{Discussion and Conclusion}

FTMH with Coats disease has rarely been reported [3-8] and the pathogenesis of FTMH is unknown. To our knowledge, this is the first report of a patient with Coats disease who developed FTMH.

In previous reports, OCT findings in FTMH with Coats disease have included 1 case with thin membranous tissue [3], 1 case with epiretinal membrane (ERM) [6], and 2 cases with no 


\section{Case Reports in Ophthalmology}

Hashimoto et al.: Full-Thickness Macular Hole Formation and Coats Disease

macular traction $[4,5]$. In the present case, OCT showed no sign of vitreomacular traction, ERM.

Kumar et al. [6] described a role for tangential traction induced by ERM in the etiology of FTMH. They performed a 25 -gauge pars plana vitrectomy and ILM peeling. After surgery, their patient was found to have functional preservation with good visual and anatomic outcomes. In our case, there was no ERM and no posterior vitreous detachment but only tangential traction of internal limiting membrane.

Kumar et al. [4] also reported that peripheral vascular leakage may lead to macular edema even though the vascular telangiectasia is located in the retinal periphery. In our case, serous macular detachment occurred after intravitreal anti-VEGF therapy. This may have been secondary changes in the vascular telangiectasia located in the retinal periphery.

The causes or mechanisms of secondary FTMH have been reported, such as retinal vitreous surgery, diabetic edema, retinal pigment epithelial detachment, and intravitreal injection of anti-VEGF [7-10]. Our patient had received intravitreal anti-VEGF therapy; however, FTMH occurred at least 1 year after the anti-VEGF injections, and thus the effect might be negligible in our case.

Brazitikos and Stangos [11] described macular hole formation in diabetic retinopathy with concurrent cystoid macular edema. Their hypothesis was that intraretinal exudate could lead to cystoid degeneration of the retina, which might likely progress spontaneously to macular retinoschisis or even partial or complete macular hole formation. In cases where there is thinning of the inner retinal wall in serous retinal detachment, FTMH could develop after rupture of the serous retinal detachment, as a result of increased internal pressure. It is also possible that traction arising from a remnant posterior hyaloid membrane on the surface of the juxtafoveal retina may contribute to FTMH and that posterior hyaloid traction might contribute even more strongly.

There were few reports of vitrectomy for Coats disease. In eyes with extensive exudative retinal detachment, vitrectomy was performed with external subretinal fluid drainage [12]. Moreover, in case of secondary ERM [13] and FTMH [6] with Coats disease, vitrectomy was also performed.

In the present case, we speculate that peripheral vascular leakage may have caused chronic inflammation, thus later on causing tissue contraction. It is thought that repeated exacerbation and remission of chronic peripheral inflammation may cause tangential traction of ILM, subretinal detachment and retinal thinning. Application of PC to such conditions may cause further intraocular inflammation. Anti-VEGF therapy induced a temporal remission, yet injections might have enhanced tangential traction of ILM.

To our knowledge, this is the first report on a patient who developed FTMH during followup for Coats disease. In this case, the vitrectomy for FTMH with Coats disease provided good visual and anatomic outcomes.

\section{Statement of Ethics}

This research complies with the relevant guidelines for human studies and was conducted ethically in accordance with the World Medical Association Declaration of Helsinki. The study protocol was approved by the IRB of Jichi Medical University. Patient consent to review his medical records was not required by the IRB, because the analyzed data does not contain any personally identifiable data. 


\section{Case Reports in Ophthalmology}

\section{Conflict of Interest Statement}

The authors have no conflicts of interest to declare.

\section{Funding Sources}

The authors received no funding.

\section{Author Contributions}

Management and interpretation of the data: Yusuke Arai, Shinji Makino, Yuji Inoue, Hidenori Takahashi; collection of the data: Yusuke Arai, Yuto Hashimoto; preparation, review, or approval of the manuscript: Yusuke Arai, Shinji Makino, Yuji Inoue, Hidenori Takahashi, and Hidetoshi Kawashima.

\section{References}

1 Shields JA, Shields CL, Honavar SG, Demirci H, Cater J. Classification and management of Coats disease: the 2000 proctor lecture. Am J Ophthalmol. 2001 May;131(5):572-83.

2 Shields JA, Shields CL, Honavar SG, Demirci H. Clinical variations and complications of Coats disease in 150 cases: the 2000 Sanford Gifford Memorial Lecture. Am J Ophthalmol. 2001 May;131(5):561-71.

3 Ioannidis AS, Liasis A, Sheldrick J, Snead M, Nischal KK. Lamellar macular hole as the presenting feature in a child with Coats' disease. J Pediatr Ophthalmol Strabismus. 2005 Nov-Dec;42(6):378-9.

4 Kumar V, Goel N, Ghosh B, Raina UK. Full-thickness macular hole and macular telangiectasia in a child with Coat's disease. Ophthalmic Surg Lasers Imaging. 2010;41 Online:e1-3.

5 Wong SC, Neuwelt MD, Trese MT. Delayed closure of paediatric macular hole in Coats' disease. Acta Ophthalmol. 2012 Jun;90(4):e326-7.

6 Kumar V, Kumar P, Garg G, Damodaran S. Vitrectomy for full-thickness macular hole in adult-onset Coats' disease. Indian J Ophthalmol. 2017 Nov;65(11):1246-8.

7 Amemiya T, Yoshida H. Macular hole in diabetic maculopathy. Ophthalmologica. 1978;177(4):188-91.

8 Lee SH, Park KH, Kim JH, Heo JW, Yu HG, Yu YS, et al. Secondary macular hole formation after vitrectomy. Retina. 2010 Jul-Aug;30(7):1072-7.

9 Nagaoka K, Inoda S, Takahashi H, Arai Y, Inoue Y, Takizawa Y, et al. Case of giant macular hole progression after rupture of a giant retinal pigment epithelial detachment. Case Rep Ophthalmol. 2019 Jun;10(2):195-9.

10 Querques G, Souied EH, Soubrane G. Macular hole following intravitreal ranibizumab injection for choroidal neovascular membrane caused by age-related macular degeneration. Acta Ophthalmol. 2009 Mar;87(2):235-7.

11 Brazitikos PD, Stangos NT. Macular hole formation in diabetic retinopathy: the role of coexisting macular edema. Doc Ophthalmol. 1999;97(3-4):273-8.

12 Kusaka S. Surgical Management of Coats Disease. Asia Pac J Ophthalmol (Phila). 2018 May-Jun;7(3):156-9.

13 Kumar P, Kumar V. Vitrectomy for epiretinal membrane in adult-onset Coats' disease. Indian J Ophthalmol. 2017 Oct;65(10):1046-8. 


\section{Case Reports in Ophthalmology}
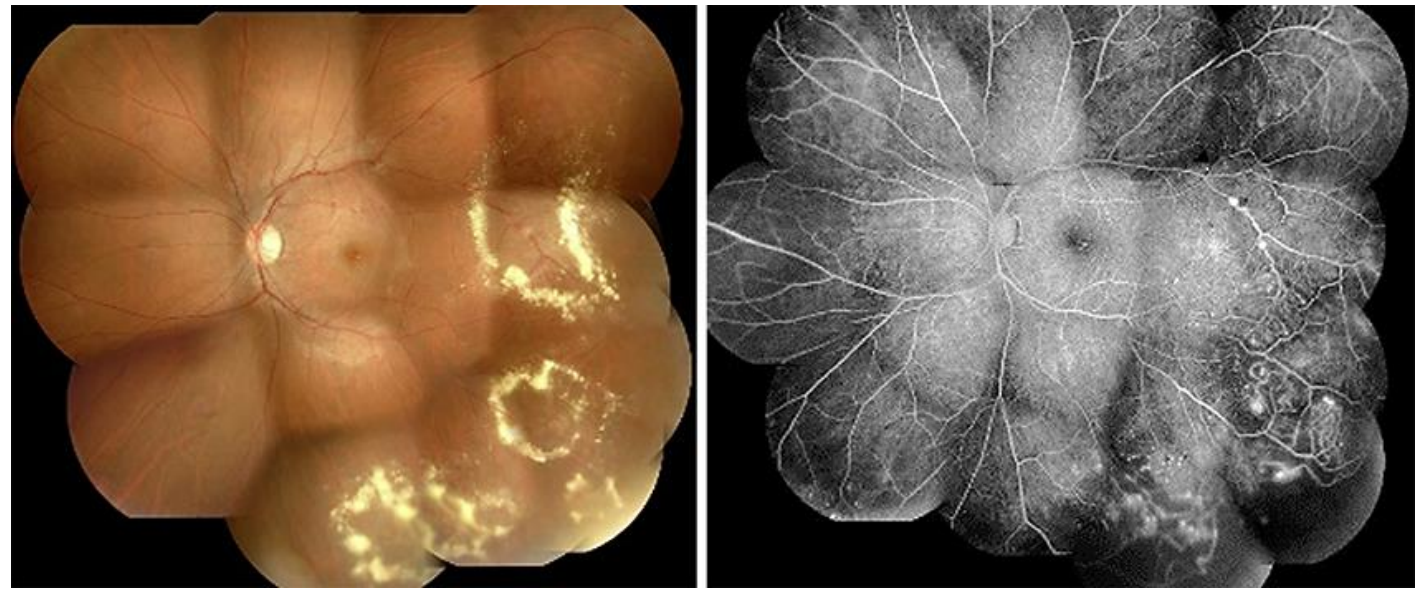

Fig. 1. Fundoscopy at the first visit showing yellowish subretinal exudates with overlying telangiectatic retinal vessels in the temporal periphery of the left eye fundus. Fluorescein angiography reveals diffuse temporal peripheral vascular leakage.
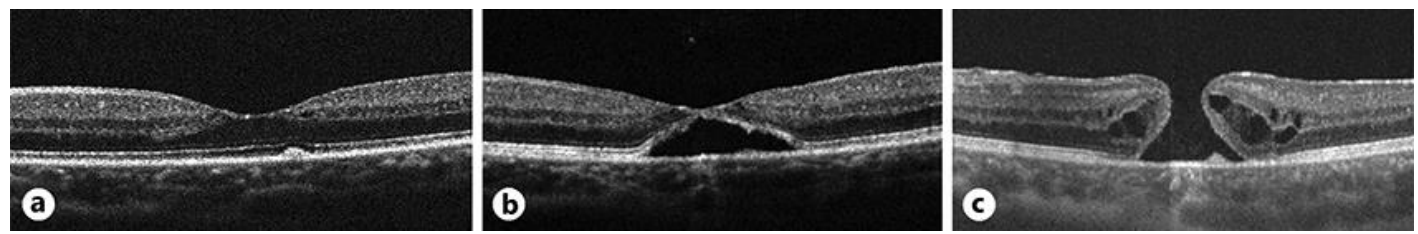

Fig. 2. a Optical coherence tomography (OCT) at the first visit showing absence of epiretinal membrane and exudate. Findings are highly indicative of internal limiting membrane and retinal deposits. b OCT at 30 months after the first visit showing tensioned internal limiting membrane (ILM) serous macular detachment, and retinal thinning. c OCT at 36 months after the first visit showing full-thickness macular hole (FTMH) formation. 


\section{Case Reports in Ophthalmology}

Hashimoto et al.: Full-Thickness Macular Hole Formation and Coats Disease
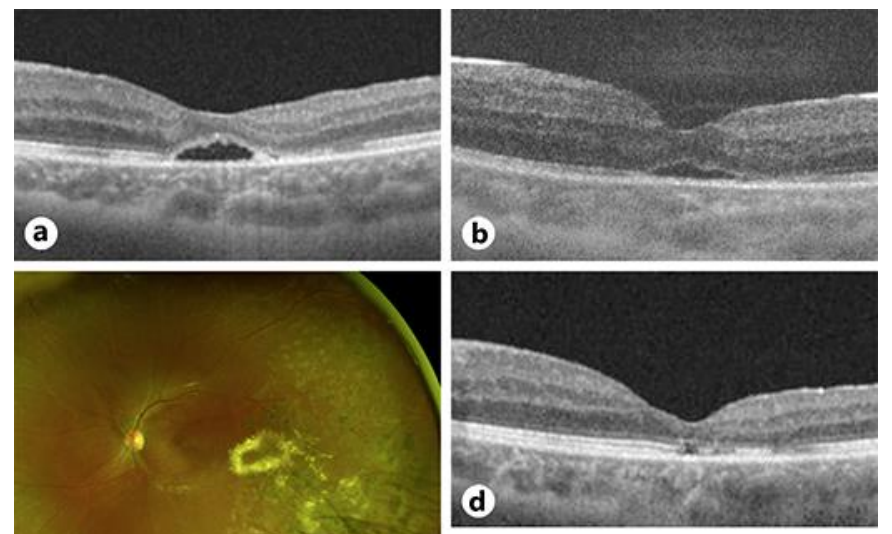

c

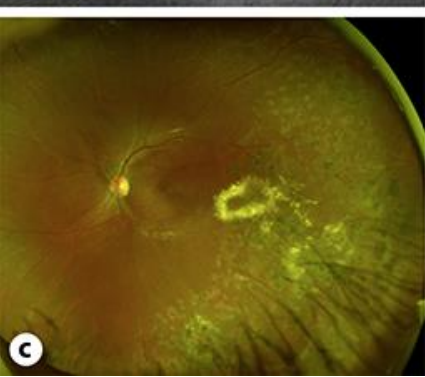

Fig. 3. a OCT at 4 days after vitrectomy showing closure of the full-thickness macular hole (FTMH). However, the serous macular detachment persists. b OCT at 1 month after vitrectomy showing serous macular detachment with FTMH closure. c Fundoscopy at 6 months after vitrectomy showing the subretinal exudate had gradually disappeared. $\mathbf{d}$ OCT at 6 months after vitrectomy showing serous macular detachment has almost disappeared; visual acuity improved to $20 / 20$. 